\title{
A new hope: introducing comprehensive sex education to a Christian secondary school in Uganda after repeal of parliamentary ban
}

\author{
Jaclyn A Boozalis', Margaret H Nakibuule ${ }^{2}$, Janet P Realini ${ }^{3}$, Jason Rosenfeld ${ }^{1}$ \\ 1 University of Texas Health San Antonio (UTHSA), San Antonio, Texas, USA, ${ }^{2}$ Hope Comprehensive High School, Masaka, Uganda, 3 Healthy Futures \\ of Texas (HFT), San Antonio, Texas, USA \\ Keywords: school curriculum, sexually transmitted infections, adolescents, uganda, sex education \\ https://doi.org/10.29392/001c.12528
}

\section{Journal of Global Health Reports}

Vol. 4, 2020

\begin{abstract}
Background
One quarter of Ugandan girls aged 15-19 years have become pregnant, and by age 50, one quarter of women report experiencing a sexually transmitted infection (STI). Adolescent pregnancies pose health challenges because teenage mothers are constrained in their future pursuit of education and have an increased likelihood of adverse pregnancy outcomes. Uganda lifted a parliamentary ban on sexual education beyond abstinence in May 2018. This study aimed to introduce and evaluate a comprehensive sex education curriculum to prevent pregnancies and sexually transmitted infections at a secondary school in Uganda.
\end{abstract}

\begin{abstract}
Methods
Over three weeks in June 2018, our team implemented a comprehensive reproductive health education curriculum called 'big decisions' in a secondary school in Uganda. Hope Comprehensive High School is a Christian school in Masaka, Uganda with approximately 400 students. We trained 15 teachers and co-facilitated the curriculum with a pilot group of 30 students. We measured student demographics and knowledge/attitudes toward sex, relationships, STIs, and contraception before and after the program.
\end{abstract}

\section{Results}

There was a statistically significant improvement in student knowledge about pregnancy, contraceptives, and STIs. We also noted an increase in positive attitudes toward condom use.

\section{Conclusions}

Engaging the faculty and staff with an appropriate and fact-based curriculum was crucial to the success of the project. Myths on contraceptive education and condoms were significantly debunked within the pilot group. This project demonstrates the viability of a non-faith based sex education curriculum in a Christian Ugandan secondary school.

According to the Uganda's 2016 Demographic and Health Survey, $25 \%$ of girls aged $15-19$ years had become pregnant. Per this survey, $14 \%$ of men and $25 \%$ of women reported having a sexually transmitted infection (STI) before age 50, and over a quarter of these people did not seek medical treatment. ${ }^{1}$ Adolescent pregnancy poses a health challenge because teenage mothers are constrained in their future pursuit of education, have an increased likelihood of adverse pregnancy outcomes, and children born to younger mothers are at higher risk for illness and death. ${ }^{2}$ The issues of teenage pregnancy and spread of STIs are multifactorial, and in large part due to lack of comprehensive sexual education. ${ }^{3,4}$ Ugandan sexual education is embedded in the information students receive at school from their teachers and health workers, which historically emphasized abstinence-only and contraceptive-discouraging messages. ${ }^{4}$
Sex education in Uganda has faced many challenges. In 2016, the Ugandan government decreed a parliamentary ban on comprehensive sex education beyond "abstinence only.” During this time, abstinence-only sex education in Uganda was ineffective, facilitating the dissemination of misinformation on the effectiveness of condoms and negatively affecting the self-esteem of youth. The director of the Family Life Network, an organization largely funded and supported by the Ugandan government, factitiously promoted that "the failure rate of condoms used against HIV is 20 percent." In the early 2000 s, the director also drafted secondary school materials for the Presidential Initiative on AIDS Strategy for Communication to the Youth (PIASCY) that falsely claimed that condoms contain microscopic pores that are permeable to HIV. ${ }^{5}$ Further, a recent study of Ugandan students found that they did not see protected sex 
as an alternative to abstinence because they perceived that condoms barely reduced the risk of contracting HIV or becoming pregnant. ${ }^{4}$ Abstinence-only education has also contributed to damaging students' sense of self-worth; if a student has sex, they view their self-worth as significantly lower and perceive that they have almost nothing left to lose when their virginity is gone, leading to risky sexual behavior. ${ }^{6}$ As of May 17, 2018, the first lady/minister of education officially launched the national sexuality education framework, effectively repealing the bans created in 2016. The framework is similar to other comprehensive sexual education curricula in that it discusses providing a "holistic" sexual education and allowing young people to make decisions for themselves, but differs in that its number one principle is "God-fearing" and it does not mention condom use or contraceptives. ${ }^{7}$

In June 2017, our team of medical students from the University of Texas Health San Antonio (UTHSA) supported by the Center for Medical Humanities and Ethics was working in Masaka, Uganda. While in-country, our partners, US2UGANDA4LIFE, informed us that they had seen an increase in pregnancies amongst female students and an increased incidence of STIs amongst both males and females at Hope Comprehensive High School in Masaka over the previous year. In response, our team conducted an assessment at the school and determined that sexual education was a priority. ${ }^{8}$ In the summer of 2018 , Hope School invited our team to test the implementation of a comprehensive sexual education curriculum with a selection of Hope School staff and students. The course used was 'big decisions', a curriculum developed by Janet Realini, MD, MPH, to reduce teen pregnancies and risk for STIs in South Texas adolescents. ${ }^{9}$

\section{METHODS}

The UTHSA institutional review board determined this project was non-regulated research per federal regulations (Protocol number: HSC20180254N).

We used 'big decisions' to teach sexual education to a pilot group of 30 students at Hope School. 'big decisions' is an abstinence plus curriculum, which combines messages focused on "vigorous abstinence promotion and positive contraceptive information." 9 This curriculum addresses many of the aforementioned determinants of adolescent pregnancy and infection with HIV. It consists of ten lessons, each requiring about one hour to teach, which include: 1) rules of the game: forming a respectful group, 2) goals and dreams: my future, 3) relationships and romance: what is healthy? 4) anatomy and reproduction: how it works, 5) abstinence: decisions to wait, 6) a clear "NO": my limits, 7) contraception: pregnancy at a good time for you, 8) sexually transmitted infections, including HIV/AIDS, 9) staying healthy and on track: defending my limits, 10) overcoming challenges: 'big decisions'. One of the primary purposes of the 'big decisions' curriculum is to deliver facts to students about their health, and to stimulate discussions where each individual can ultimately make resilient choices for themselves.

Facilitation of the curriculum focuses on creating a safe space, inclusivity, and being trauma-informed. A study con-
HFT trains 8 UTHSA students to facilitate Big Decisions in San Antonio, TX

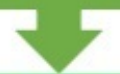

UTHSA students train 15 teachers at Hope School in Masaka, Uganda

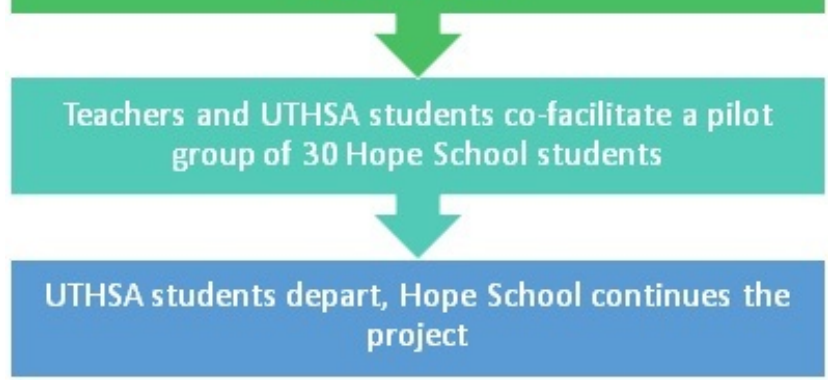

Figure 1. Flow chart for method of training and introduction of project.

ducted on 788 U.S. inner-city 9th-grade students using preand post-' big decisions' intervention surveys demonstrated that participation in the program was associated with positive changes in student's attitudes towards abstinence, STIs, condoms, contraception, and avoiding sexual pressures. 10

Members of our team received 18 hours of 'big decisions' facilitation training from a Healthy Futures of Texas Health (HFT) education program manager (Figure 1). Upon arriving in Uganda, our team administered the same training to Hope School teachers over two days.

After completion of teacher training, each of our team members paired up with Hope teachers to facilitate a lesson for a pilot group of 30 Hope students. The pilot group consisted of 14 girls and 16 boys between the ages of 14 and 18 . School administrators selected students who were leaders (eg, prefects, time keepers) to pave the way for other students, and did not single out or target students based on their sexual activity or behavior.

The curriculum was taught over one week. We administered pre- and post-intervention written surveys in order to assess changes in knowledge and attitudes about sex-related topics. The Student Participant survey was based on selections from a survey designed by John Cleland titled "illustrative questionnaire for interview-surveys with young people." 11

After completion of the pilot program, we met with Hope School staff to create a timeline for the continued implementation of the curriculum and administration of surveys during the 2018-2019 academic school year. We utilized Microsoft Excel (Microsoft Inc, Seattle, WA, USA) to manage raw data and IBM SPSS Statistics v25 (IBM, Armonk NY, USA) to analyze survey data for significant improvements in knowledge and attitudes toward sex and condom use.

\section{RESULTS}

$\chi^{2}$ tests show that the percentage of correct responses on 
Table 1. Percentage of correct responses on knowledge-based questions in pre- and post-survey*

\begin{tabular}{lccc}
\hline True/False Question & Pre-percent correct & Post-percent correct & $\mathbf{X}^{2}$ \\
\hline T/F: A woman may become pregnant at first sexual intercourse & $41.0 \%$ & $59.0 \%$ & 0.016 \\
\hline T/F: It is currently possible to cure AIDS & $37.5 \%$ & $62.7 \%$ & $0.005 \dagger$ \\
\hline T/F: Condoms protect against HIV & $37.8 \%$ & $62.2 \%$ & $0.040 \dagger$ \\
\hline T/F: Condoms protect against STIs & $31.4 \%$ & $68.6 \%$ & $0.003 \dagger$ \\
\hline
\end{tabular}

*The correct response to each True/False question is in bold.

tFischer's-Exact Test

the knowledge questions improved significantly from pre to post-survey (Table 1). Further, the proportion of students who held positive attitudes toward suggesting condom use to a partner increased. Students who agreed that a boy can suggest to a girl that they use a condom increased from $73.3 \%$ to $93.1 \%$, and students consistently agreed that a girl can suggest that a boy use a condom (from $86.7 \%$ to $89.7 \%$ ). The number of students who were confident that they could use a condom increased by $24.7 \%$ (from $46.7 \%$ to $71.4 \%$ ). Students who reported that the most suitable method of contraception for young people is abstinence increased by $32.4 \%$ (from $40.0 \%$ to $72.4 \%$ ).

\section{DISCUSSION}

The cornerstone to the successful reception of the curriculum was early engagement of the faculty and staff at Hope School. We mailed a hard copy of the curriculum to the school so they had an opportunity to review it prior to our arrival. Administrators were initially concerned with teaching about contraceptives because they believed it would encourage students to have sex. However, after a thorough discussion with faculty and reassurance from our team, we set goals and agreed to use the entire 'big decisions' curriculum. Notably, the number of students reporting abstinence as the most suitable method of contraception for young people increased from $40 \%$ to $73 \%$. This result assuaged the staff's fears and when we debriefed with the director of the school, he requested that we update the program to include a condom demonstration during the contraceptive lecture. We were able to address and overturn the perception amongst staff that learning about contraceptives would encourage students to have more sex, and able to join forces with the faculty at Hope School to educate the students on these topics.

This work is significant because it begins to address the false myths about condoms spread by the Ugandan Director of the Family Life Network. Spread of misinformation is dangerous; when students believe that condoms are ineffective at protecting against STIs, including HIV, they are more inclined to have unsafe sex. This misconception was prominent at Hope School, as $73.3 \%$ of our sample disagreed that condoms were are an effective way of protecting against the spread of HIV/AIDS before the start of the program. The 'big decisions' curriculum has been reviewed for medical accuracy, and served as an effective way to correct these misconceptions.

It is possible to teach sexual education separately from religion in Uganda. One of the pillars of 'big decisions' facilitation is to remain values-neutral i.e., for facilitator to keep their personal biases in check for the sake of student learning and discussion. The head pastor of Hope School, Matthew Kijoba, was actually the most insistent about faculty remaining values-neutral. This project demonstrates that a non-faith based sexual education curriculum is viable in a faith-based Ugandan school, and is appropriate for both students and staff.

This study is limited in that we are unable to assess the consequential behavioral measures for the validation of the effectiveness of this study in producing better sexual health outcomes. We were only able to survey the pilot group of thirty students, which is a small sample out of the hundreds of students at Hope School. The data may also not be reflective of Hope Comprehensive High School as a whole due to student selection. Students were selected by the director based on leadership and maturity, so that these students might set an example and share what they have learned from 'big decisions' with students who would complete the program later in the year.

Acknowledgements: Center for Medical Humanities and Ethics (CMHE) for providing and managing the global health program at University of Texas Health San Antonio (UTHSA), which included coordinating training sessions from Healthy Futures of Texas (HFT) for Kisoboka Uganda Team. Hope Comprehensive High School for coordinating this program with us and for their integral role as community partners and continued implementation. Kisoboka Uganda Team of UTHSA for their hard work and dedication to implementing this project. US2UGANDA4LIFE for bringing the community need to our attention.

Disclaimer: The views expressed in the submitted article are our own and not an official position of the UTHSA or HFT.

Funding: Student travel for completion of the project was funded by UTHSA and fundraising by the Kisoboka Uganda Team.

Authorship contributions: JAB: primary author, principal 
investigator, developer of study protocol, data collector, data management and analysis. MHN: survey editor, onsite program coordinator, community partner. JPR: program design, research design, results interpretation. JR: faculty advisor, manuscript editor, data analysis and interpretation, training coordinator.

Competing interests: The authors completed the Unified Competing Interest form at www.icmje.org/coi_disclosure.pdf (available upon request from the corresponding author), and declare no conflicts of interest.

Correspondence to:

Jaclyn Boozalis, BA

810 Champions Row

Victoria, TX 77904

USA

Boozalis@livemail.uthscsa.edu 


\section{REFERENCES}

1. Uganda Demographic and Health Survey 2016: Key Indicators Report. In: Uganda Bureau of Statistics TDP, USAID, UNICEF, UNFPA, UBOS. Kampala, Uganda; 2016.

2. Research DoRHa, ed. Adolescent Pregnancy: Fact Sheet. In: Research DoRHa. Geneva: World Health Organization Newsroom; 2014.

3. Yakubu I, Salisu WJ. Determinants of adolescent pregnancy in sub-Saharan Africa: A systematic review. Reprod Health. 2018;15:15. doi:10.1186/s1297 8-018-0460-4

4. de Haas B, Hutter I, Timmerman G. Young people's perceptions of relationships and sexual practices in the abstinence-only context of Uganda. Sex Education. 2017;17:1-15. doi:10.1080/14681811.201 $\underline{7.1315933}$

5. Cohen J, Tate T. The Less They Know, the Better: Abstinence-Only HIV/AIDS Programs in Uganda. Reproductive Health Matters. 2006;14:174-178. doi:1 0.1016/S0968-8080(06)28249-1

6. van de Walle E, van de Walle F. Birthspacing and Abstinence in Sub-Saharan Africa. International Family Planning Perspectives. 1988;14:25-26. doi:1 $\underline{0.2307 / 2947654}$
7. Sports MoEa, ed. National Sexuality Education Framework. In: Kampala, Uganda: Republic of Uganda; 2018.

8. Boozalis J, Kazen K, Amuneke-Nze C, Russell T, White W, Bishop T, et al. Lessons learned: A slapdash sexual education intervention for adolescent girls at a secondary boarding school in Masaka, Uganda. [Poster]. 2018.

9. Realini JP. Big Decisions: Making Healthy, Informed Choices about Sex. San Antonio, Texas, USA: Healthy Futures of Texas; 2016.

10. Realini JP, Buzi RS, Smith PB, Martinez M. Evaluation of "big decisions" ": An abstinence-plus sexuality curriculum. J Sex Marital Ther. 2010;36:313-326. doi:10.1080/0092623X.2010.488113

11. Cleland J. Illustrative Questionnaire for Interview-Surveys with Young People. Asking Young People About Sexual and Reproductive Behaviors Illustrative Core Instruments. Geneva: World Health Organization; 2001. 